\title{
Sistemas de alocação de recursos a prestadores de serviços de saúde - a experiência internacional
}

\author{
Resource allocation systems for health service providers \\ - the international experience
}

Maria Alícia Dominguez Ugá ${ }^{1}$

${ }^{1}$ Escola Nacional de Saúde Pública Sérgio Arouca, Fundação Oswaldo Cruz. Rua Leopoldo Bulhoes 1480/ 709, Manguinhos. 22241210 Rio de Janeiro RJ. domingue@ensp.fiocruz.br

\begin{abstract}
This article presents the traditional ways of allocating resources to health service providers and focuses on the presentation and discussion of alternative experiences found in the international context. It also shows the current trends in the OECD countries, involving the adoption of mixed systems or performance-related bonuses, the latter being predominantly referred to the effects on the health of the population, i.e. the effectiveness of the health services. It further stresses the tendency to adopt resource allocation systems that are differentiated according to the level of care provider: to primary care centers, responsible for the health of the population of a given territory, a per capita adjusted for risk factor is granted (or, in some cases, resource allocation defined for lines of care), while in other cases hospitals are either paid according to a performance-adjusted global budget or through prospective payment per procedure.
\end{abstract}

Key words Resource allocation, Service provider payment systems
Resumo O artigo apresenta as formas tradicionais de alocação de recursos a prestadores de serviços de saúde e se concentra na apresentação e discussão de experiências alternativas encontradas no contexto internacional. Aponta, ainda, as tendências atuais formuladas nos países da OECD, que consistem na adoção de sistemas mistos ou complementados pelo ajuste por desempenho, sendo este predominantemente referido a resultados sobre a saúde da população, ou seja, à efetividade dos serviços de saúde. Ainda, destaca-se uma tendência a adotar sistemas de alocação de recursos diferenciados, segundo o nível de atenção do prestador: aos centros de atenção primária responsáveis pela saúde da população de um dado território, corresponde a alocação de recursos per capita ajustada por risco (ou, em alguns casos, por linhas de cuidado), enquanto os hospitais são em alguns casos remunerados por orçamento global ajustado por desempenho e, em outros, por pagamento prospectivo por procedimento.

Palavras-chave Alocação de recursos, Sistemas de pagamento a prestadores 


\section{Introdução}

Já nos anos setenta apontavam Wennberg e Gittelsohn ${ }^{1}$ que "o volume e custos dos tratamentos hospitalares em uma dada comunidade têm mais relação com o número de médicos que lá atuam, suas especialidades e os procedimentos que eles preferem do que com a saúde da população”. Embora eles estivessem, naquele momento, referindo-se à realidade estadunidense, provavelmente em qualquer sociedade capitalista, ainda hoje, essa afirmação poderia ser reiterada.

Nesse contexto e, particularmente, no âmbito das reformas dos sistemas de saúde dos anos noventa, movidas fundamentalmente por critérios de ajuste macroeconômico ${ }^{2}$, os sistemas de alocação de recursos (ou "modos de pagamento", como denominado na literatura internacional) a hospitais e prestadores de serviços ambulatoriais passam a ser vistos como um instrumento de contenção da expansão exponencial dos gastos em saúde, tanto pelos sistemas públicos como por sistemas privados que gerem redes de serviços, no modelo de atenção gerenciada ( $m a-$ naged care).

Para além dessa questão, a necessária capacidade de condução do sistema de saúde a partir de redes integradas de serviços requer instrumentos de regulação dos prestadores de serviços de saúde, tais como modelos de contratualização entre o gestor público e prestadores e, também, novos sistemas de remuneração aos prestadores de serviços que superem o mero pagamento por serviços previamente prestados.

Ainda, está cada vez mais presente nos debates sobre a gestão de sistemas de saúde a necessidade qualificar o gasto em saúde, mediante o uso da avaliação econômica para a incorporação de tecnologias: antes de incorporar novas tecnologias médicas é necessário conhecer a relação entre o seu custo adicional em relação às que lhe antecedem e a efetividade adicional que elas trazem. Segundo o BMJ Clinical Evidence Hand$b_{o o k}^{3}$ apud Maynard ${ }^{4}$, a efetividade de mais da metade da produção de procedimentos médicoassistenciais carece de comprovação. Ainda, como afirma este último autor, a incerteza que prevalece em relação à efetividade de grande parte dos procedimentos introduz uma importante variação na prática clínica, de forma que, nas palavras desse autor, "na maior parte dos sistemas de saúde há evidência de que intervenções provadamente custo-efetivas não são realizadas nos pacientes e, o que é mais grave, intervenções inadequadas são feitas nos pacientes sem nenhum benefício e com possível dano". Nesse contexto e no sentido de dotar de maior racionaliade técnica a prática médico-assistencial, as formas de contratualização e de alocação de recursos a prestadores hospitalares e ambulatoriais também têm um papel a cumprir, de forma a induzí-los a adotar práticas e tecnologias mais custo-efetivas e seguras para os pacientes.

\section{As formas tradicionais de alocação de recursos aos prestadores de serviços}

Como apresentado em Ugá ${ }^{5}$ os métodos de alocação de recursos a prestadores de serviços hospitalares ou ambulatoriais podem ser classificados a partir do momento em que se dão essas transferências financeiras: se antes da efetiva produção de serviços (alocação ex-ante) ou se depois e em função do volume e tipo da produção apresentada por cada prestador (alocação ex-post).

No primeiro grupo (alocação ex-ante), encontram-se o orçamento global e a alocação de recursos por capitação (ou per capita); no segundo (alocação ex-post), estão os sistemas que se constituem, estes sim, na remuneração por serviços previamente prestados, como é o caso do pagamento por ato médico, do pagamento por diária hospitalar e do pagamento prospectivo por procedimento. Esses cinco métodos são apresentados sucintamente a seguir.

\section{Sistemas de alocação de recursos ex-ante:}

\section{A alocação de recursos \\ por orçamento global}

O sistema de alocação de recursos por orçamento global consiste em repasses periódicos de um montante anual de recursos definido através de programação orçamentária elaborada pela unidade de saúde para o período correspondente e negociada com o órgão financiador.

Trata-se de um sistema no qual, embora o montante de recursos esteja formalmente calculado em base à produção prevista para o período em questão (geralmente um ano), as transferências de recursos não estão vinculadas à efetiva produção de serviços. Assim, se por um lado ele possibilita ao órgão financiador uma previsão dos seus gastos e à unidade de saúde uma previsibilidade de suas receitas (desde que não haja o contingenciamento dos recursos e, portanto, atraso na liberação dos mesmos, como tem ocorrido no Brasil com frequência, principalmente a partir de meados dos anos noventa, anos do ajuste macro-econômico), este método de alocação de 
recursos não traz em si - e em sua forma clássica - nenhum incentivo ao bom desempenho do prestador de serviços, nem em termos quantitativos, nem qualitativos.

\section{A alocação de recursos por capitação}

Esta forma de alocação pressupõe a existência de um sistema de saúde regionalizado e hierarquizado, no qual a cada unidade prestadora de serviços esteja vinculada uma população adscrita, previamente definida. Assim, a cada prestador corresponde um montante de recursos (periódico - geralmente anual) que equivale ao número de indivíduos a ele adscritos multiplicado por um valor per capita. Este valor unitário é frequentemente ajustado por risco, tomando-se em conta, pelo menos, o sexo e a idade da população adscrita.

O sistema em questão é utilizado há décadas para a alocação de recursos aos médicos generalistas (general practitioners - GP) que atuam como porta de entrada no Sistema Nacional de Saúde Inglês e foi, na reforma dos anos noventa, estendido para os gestores do sistema em nível microrregional, isto é, os GP-funholders (gestores de recursos públicos) e as autoridades sanitárias locais.

$\mathrm{Na}$ ausência de um sistema de avaliação e controle sistemático, este método de alocação de recursos pode levar à subprodução de serviços, tendo em vista que o valor recebido pelo prestador independe da produção, sendo atrelado ao número de pessoas adscritas - e não ao número de pacientes tratados. Nesse sentido, nas reformas dos noventa introduziu-se no Reino Unido a concorrência dos GP por clientela, desvinculando-se a adscrição ao local de moradia da população. Entretanto, da mesma forma que o orçamento global, apresenta a virtude da previsibilidade do gasto do órgão financiador e da receita do prestador.

\section{Sistemas de alocação de recursos ex-post:}

Estes constituem modalidades de pagamento por serviços previamente prestados. $\mathrm{O}$ valor a ser pago pode ser calculado a priori (prospectivamente) ou a posteriori, como é o caso do pagamento itemizado e do pagamento por diária hospitalar, apresentados a seguir.

\section{O pagamento por ato médico} (ou itemizado)

Este método vincula o pagamento a serviços previamente efetuados - mais especificamente, ao tratamento de cada paciente - e consiste na remuneração discriminada de cada item que compõe o procedimento efetuado (materiais e medicamentos, honorários profissionais, diárias hospitalares e serviços intermediários, tais como exames, dentre outros).

Assim, o valor mensal correspondente a cada prestador equivale ao somatório dos serviços intermediários e finais prestados a cada paciente, multiplicados por seus respectivos valores. Por sua vez, estes últimos são, em alguns casos, negociados previamente entre o órgão financiador e os prestadores e, em outros - menos frequentes, definidos a posteriori a partir dos custos observados (aos quais se adiciona, no caso do setor privado, sua margem de lucro).

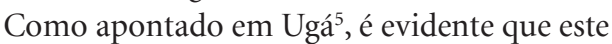
sistema estimula a sobreutilização de serviços intermediários, principalmente os que proporcionam markups mais elevados e, portanto, afeta negativamente a qualidade da atenção médicoassistencial, seja porque pode gerar episódios iatrogênicos, seja por submeter o paciente a desconfortos evitáveis e indesejáveis. Além disso - e em função desse fenômeno - tende a gerar um aumento desnecessário no custo da assistência médica.

\section{O pagamento por diária hospitalar}

Trata-se evidentemente de um método que remunera exclusivamente as internações hospitalares, não sendo aplicável aos serviços ambulatoriais. Através dele, o valor correspondente periodicamente a cada hospital equivale ao número de diárias (ou seja, ao somatório do tempo de permanência dos pacientes internados no período em questão) multiplicado por um valor unitário atribuído a cada diária.

O valor da diária é definido a partir do seu custo médico, incluindo ou não os honorários médicos. Em grande parte dos países que adotam este sistema de pagamento, tendo em vista as enormes disparidades de custo de cada tipo de internação (que não só depende do tempo de permanência, mas também dos insumos utilizados, tais como exames complementares e medicamentos usados no período da internação), são definidos valores diferenciados segundo a complexidade do hospital e a clínica (médica, cirúrgica, UTI, etc.). 
Vale observar, entretanto, que através deste mecanismo de remuneração os hospitais não são remunerados pelo custo efetivo de cada paciente tratado, o qual pode levar à segregação de alguns pacientes, segundo seja seu quadro nosológico.

Por outro lado, tendo em vista que o hospital recebe por diárias, este método induz ao prolongamento desnecessário do tempo de permanência, tendo em vista que o custo da internação de cada paciente tende a ser decrescente com o tempo, pois a maior parte dos exames complementares de diagnóstico e o ato cirúrgico são geralmente efetuados no início da internação. Em função disso, em alguns países são estipulados valores da diária que decrescem com o tempo de permanência.

De todas formas, é um método de pagamento altamente insatisfatório pelos motivos acima apontados e, atualmente, na maior parte dos países que o adotavam na sua forma pura (França e Alemanha, dentre outros), esta forma de remuneração foi associada a outras, constituindo sistemas mistos, como será discutido em outra seção deste documento.

\section{O sistema de pagamento prospectivo por procedimento}

Através deste sistema, o prestador de serviços de saúde é remunerado por episódio de tratamento - no caso do hospital, por internação com valores diferenciados segundo o diagnóstico do paciente. Trata-se de um método prospectivo (e não retrospectivo), porque os valores atribuídos a cada internação são definidos previamente, embora variem segundo o diagnóstico. Assim, o sistema estabelece, na realidade, uma série de preços pré-fixados das internações, por tipo (isto é, o diagnóstico) do paciente. Estes preços independem, portanto, do custo efetivo de cada paciente tratado no episódio de sua internação.

Vale notar que, para ser administrável, tendo em vista a infinidade de diagnósticos (diagnóstico principal e secundário, comorbidades) e de características do paciente (idade), este método de pagamento requer indispensavelmente um sistema de classificação dos pacientes, em grupos homogêneos - fundamentalmente do ponto de vista do custo. Nesse sentido, o primeiro país que adotou o sistema de pagamento prospectivo por procedimento, isto é, os EUA, o fez depois de contar com um sistema de classificação bastante robusto de pacientes, que estabeleceu os Diagnostic Related Groups - DRG (Grupos Relacionados ao Diagnóstico). Este sistema de classificação tem por objetivo subsidiar não só a gestão econômica (in- teressada no custo das internações) mas também a gestão clínica (interessada no perfil clínico dos casos tratados) e, nesse sentido, constituiu grupos que se pretenderam homogêneos do ponto de vista do custo, definidos da seguinte forma ${ }^{5}$ :

- em primeiro lugar, as altas hospitalares são classificadas a partir do diagnóstico principal em grupos de Principais Categorias Diagnósticas (baseados no sistema orgânico afetado e/ou na especialidade médica responsável pelo tratamento);

- a seguir, as altas relativas a tratamentos clínicos (não cirúrgicos) são subdivididas em categorias baseadas no seu diagnóstico principal, enquanto os casos cirúrgicos são agrupados segundo os procedimentos efetuados;

- em um terceiro momento, esses grupos são reagrupados segundo a intensidade de utilização de recursos e, portanto, o seu custo;

- finalmente, a classificação definitiva das altas hospitalares se dá através da constituição de subgrupos clínicos ou cirúrgicos, configurados a partir de outras características do paciente (idade, diagnóstico secundário, comorbidades e complicações, dentre outras) que afetam o tempo de permanência do paciente no hospital e, portanto, o seu custo.

O sistema de pagamento prospectivo por procedimento requer, além de um método de classificação dos pacientes adequado ao custo dos tratamentos neles efetuados, um sistema de definição dos preços unitários das altas que leve em conta características do hospital que também afetam seus custos (principalmente, seus custos fixos), tais como seu nível de complexidade e o fato de ser ou não um hospital de ensino, dentre outros.

Nesse sentido, nos EUA o sistema de pagamento prospectivo se dá da seguinte forma:

- a cada grupo de DRG corresponde um determinado número de pontos, $\mathrm{P}(\mathrm{i})$;

- a cada hospital corresponde uma tarifa unitária, $\mathrm{T}(\mathrm{h})$;

- o valor pago por cada internação é igual a $\mathrm{P}(\mathrm{i}) \mathrm{x} \mathrm{T}(\mathrm{h})$, isto é, ao produto do número de pontos do DRG correspondente multiplicado pela tarifa unitária do hospital.

Além disso, estão previstos pagamentos diferenciados para pacientes outliers, que fogem do padrão estabelecido nos DRG.

Apesar desses cuidados, no sentido de estabelecer preços que levem em conta as diferenças dos pacientes (expressas em sua classificação nos DRG) e as diferenças dos custos fixos dos hospitais, segundo a seu tipo (expressas na definição das tarifas unitárias diferenciadas), inúmeros problemas foram detectados nos EUA'. 
Nesse país, a experiência mostrou que, tendo em vista que o sistema remunera os hospitais segundo valores pré-fixados, que independem do custo efetivo de cada internação, o sistema tem levado à redução dos insumos e serviços intermediários utilizados em cada internação (necessários ou não) e, assim, à alteração de suas funções de produção com o intuito de diminuírem o custo das internações ${ }^{7}$. Também foi observado o aumento das reinternações, o qual sugere que altas precoces foram dadas, com o intuito de reduzir custos e/ou gerar novas internações a serem remuneradas ${ }^{8-10}$. Outro fenômeno apontado por vários autores, dentre os quais Carter et al. ${ }^{11}$, foi o da atribuição, aos pacientes internados, de DRG que não correspondiam exatamente ao seu perfil, e sim a grupos de mais alta pontuação, no fenômeno denominado de DRG creep. Finalmente, de acordo com Guterman e Dobson $^{12}$, pode-se supor que o método de pagamento prospectivo por procedimento leve a uma mudança das prioridades dos prestadores, na qual se subordine a preocupação com a qualidade a objetivos meramente financeiros.

De todas formas, este método vem sendo progressivamente incorporado em vários sistemas de saúde (Portugal, Espanha, dentre outros), porque se espera dele a indução à contenção dos custos unitários das internações hospitalares e, assim, a redução do gasto em saúde. Da mesma forma, os sistemas de classificação de pacientes (dentre os quais o dos DRG) vêm sendo há muitos anos adotados em países como Irlanda para a construção do orçamento dos hospitais a partir do case-mix desses prestadores. O case-mix corresponde ao perfil da produção do hospital, segundo grupos de classificação de pacientes e sua respectiva frequência na produção hospitalar.

Evidentemente, nenhum modelo tradicional de alocação ou pagamento consegue conciliar a previsibilidade de receitas e despesas e a desvinculação do paciente de algum preço (como é o caso dos sistemas de alocação de recursos por orçamento global e por capitação) com o estímulo a um melhor desempenho do prestador de serviços de saúde, tanto em termos quantitativos (como o faz o pagamento prospectivo por procedimento) como qualitativos (aspecto não alcançado por nenhum dos métodos tradicionais de alocação de recursos).

$\mathrm{Na}$ busca por melhores alternativas, alguns países vêm incorporando soluções distintas, seja através de sistemas mistos, seja através do pagamento por desempenho (payment for performance), seja através de outras propostas. Esse é o objeto da seção que segue.

\section{Sistemas alternativos encontrados na experiência internacional}

Os países da OCDE têm-se debruçado nos últimos anos fundamentalmente sobre sistemas para alocação de recursos a hospitais, tendo em vista que o gasto hospitalar consumiu em 2008, em média, um terço do gasto em saúde nos países da OCDE e, em alguns países europeus, chega a representar nada menos que $70 \%$ desse gasto $^{13}$. Entretanto, abaixo são apresentadas experiências que se referem, em alguns casos, à alocação de recursos para a atenção primária.

\section{Sistemas mistos}

Alguns países, tais como Espanha, Portugal, França, Alemanha, Holanda e Suíça, dentre outros, adotaram no final dos anos noventa ou início da atual década sistemas mistos de alocação de recursos aos prestadores de serviços de saúde.

Assim, enquanto os três últimos associaram o orçamento global ao pagamento por diária hospitalar, Portugal e algumas províncias espanholas adotaram sistemas compostos por orçamento global e pagamento prospectivo por DRG.

$\mathrm{Na}$ França, por exemplo, os hospitais públicos foram nos anos noventa financiados mediante orçamento global, acrescido de um pagamento por diária, do qual $20 \%$ incidem sobre o paciente (em função disso, existe um mercado de seguros especificamente em relação a este elevado copagamento). Note-se que o valor da diária varia segundo o serviço e o tipo de hospital. Atualmente, esse país adotou o pagamento prospectivo por procedimento.

Na Alemanha, aos hospitais públicos é atribuído um orçamento global negociado com o fundo financiador; entretanto, a alocação final de recursos é realizada através de pagamento por diária hospitalar.

Por sua vez, a Holanda atribui aos hospitais públicos um orçamento global que estabelece o montante total anual a ser recebido por cada hospital, em base ao tamanho da população a ser atendida, à capacidade instalada contratada (leitos segundo especialidades) e à produção contratada (número de internações, consultas e hospital-dia). Entretanto, assim como na Alemanha, a alocação efetiva de recursos é feita através de pagamento por diária hospitalar.

Algumas províncias espanholas - como Catalunha e Valencia - adotaram no final dos anos noventa um sistema misto para a alocação de recursos aos hospitais públicos. Um orçamento global estabelece o montante total anual a ser 
recebido por cada hospital, em base ao seu casemix. Entretanto, a alocação de recursos se deu de forma composta: $65 \%$ dos recursos orçados são alocados de forma global, enquanto $35 \%$ dos mesmos são transferidos através de pagamento prospectivo por procedimento, adotando o sistema de classificação dos pacientes por DRG. Novas formas de alocação de recursos são adotadas na atualidade em algumas províncias espanholas e serão apresentadas abaixo.

Portugal foi o primeiro país europeu a adotar a composição orçamento global e pagamento por DRG: mediante orçamento global é estabelecido o montante total anual a ser recebido por cada hospital, em base ao perfil de sua produção, isto é, seu case-mix. Mas a efetiva alocação de recursos é feita crescentemente $(40 \%$ em 2001) através de pagamento prospectivo por procedimento (DRG). Atualmente, novas experiências estão sendo desenvolvidas e serão discutidas em outra seção deste documento.

\section{O pagamento por desempenho (payment for performance)}

Este modelo de alocação de recursos se insere nas reformas dos sistemas de saúde que vêm sendo implementadas em vários países europeus (Reino Unido, França, Finlândia e Polônia, dentre outros) desde o final dos anos noventa, com o intuito da redução dos custos globais do sistema de saúde e o aumento da eficiência dos prestadores, bem como da efetividade dos serviços de saúde.

Elas envolvem ações que incidem sobre a demanda de serviços de saúde e outras sobre a oferta dos mesmos, sob a égide do modelo do gerencialismo. Assim, por um lado, foram introduzidos mecanismos de gestão da demanda, tais como a introdução de médicos de porta de entrada ao sistema de saúde (gatekeepers) e de mecanismos de copagamento ou de coparticipação nos custos dos serviços. Por outro, foram desenvolvidas diversas formas de contratualização de serviços de saúde pelos gestores do sistema, às quais corresponderam modalidades de alocação de recursos segundo o desempenho do prestador, medido em termos do cumprimento de metas quantitativas e qualitativas. Mais recentemente, se destaca uma preocupação com a gestão da incorporação tecnológica e, assim, as metas - em alguns países - incluem a definição do tipo de tecnologia a ser adotada, em busca da obtenção de mais valor através dos recursos aplicados, conceito esse mais difundido na literatura em inglês sob a denominação de value for money ${ }^{14}$.
O pagamento por desempenho (payment for performance) não é, em si mesmo, um sistema de alocação de recursos ${ }^{15}$; na realidade, ele ajusta o montante de recursos a ser alocado (através de algum método tradicional, em geral por orçamento global ou por capitação e, em alguns casos, o pagamento prospectivo por procedimento) ao prestador de serviços de saúde, segundo o seu desempenho, medido em termos de indicadores que podem ser de estrutura, de processo ou de resultados e, ainda, quantitativos ou qualitativos.

No Reino Unido, no contexto do gerencialismo, foram estabelecidos contratos que continham incentivos financeiros para a melhoria do desempenho no programa de imunizações e em atividades de promoção da saúde, dentre outras ${ }^{16}$. Nesse programa, os clínicos gerais que atuam como porta de entrada no sistema (GP) podiam receber uma remuneração adicional de até 42 mil libras por médico referente ao cumprimento de metas qualitativas. Esse novo modelo de contrato entre o Estado e os GP, que contempla incentivos ao aumento da qualidade na atenção médica, associa nada menos que $25 \%$ da remuneração dos clínicos gerais ao seu desempenho. Este é medido através de 146 indicadores pertencentes aos seguintes domínios ${ }^{16}$ :

- padrões clínicos relacionados ao cuidado de pacientes;

- padrões organizacionais referentes ao registro e à informação, comunicação com os pacientes, educação e treinamento, gestão dos medicamentos, gestão clínica;

- experiência do paciente, baseada em inquéritos e consultas;

- serviços adicionais (acompanhamento da saúde infantil, de serviços de pré-natal e contracepção, dentre outros).

- cuidado holístico, baseado no desempenho no domínio clínico.

Vale mencionar que, no Sistema Nacional de Saúde inglês, o sistema de pagamento por desempenho qualitativo (pay for performance $-P 4 P$ ) foi aplicado inicialmente aos gestores de microsistemas de saúde como os GP e, a partir de 2004, aos então criados Primary Care Trusts. Ele foi aplicado, depois, também a hospitais; o orçamento destinado a cada um era ajustado segundo o cumprimento de metas de qualidade assistencial e este era aferido a partir de indicadores definidos no Quality and Outcomes Framework. Estes indicadores dizem respeito, fundamentalmente, a processos utilizados no tratamento de alguns agravos (asma, diabetes, cardiopatia isquêmica, câncer, dentre outros) definidos com a 
colaboração do National Institute for Clinical Excellence (NICE).

Este modelo vem sendo aplicado em Portugal, também para a alocação de recursos às Unidades de Saúde Familiar (USF), no âmbito da atenção primária ${ }^{17}$. Nesse país, o desempenho é aferido a partir de indicadores de acesso e utilização de serviços, adequação do cuidado, qualidade percebida pelos pacientes e eficiência econômica, no contexto de contratualização dos serviços de atenção primária, mas ainda não há experiência acumulada que permita fazer uma avaliação do seu impacto sobre o desempenho do sistema de saúde.

Isso foi possível na Inglaterra, que acumula uma experiência mais longa do pagamento ajustado por desempenho. Assim, Campbell et al. ${ }^{18}$ desenvolveram recentemente um estudo sobre os efeitos dessa forma de alocação de recursos sobre a qualidade da atenção primária nesse país. $\mathrm{O}$ trabalho compara os dois anos prévios à implementação do sistema com dois anos posteriores à mesma e mostra que os indicadores de qualidade referidos à atenção de pacientes com asma, diabetes e cardiopatia isquêmica já haviam melhorado (no caso dos dois primeiros) em período prévio ao pagamento por desempenho e continuaram exibindo melhores resultados - embora com uma melhora mais lenta - após a implantação do P4P. Diferentemente, mostra o mesmo estudo, que se observaram pioras nos indicadores de atenção à cardiopatia isquêmica. Peiróo ${ }^{19}$ comenta o artigo, levantando a hipótese de que depois de um período de melhora nos indicadores, seja mais difícil manter o ritmo de crescimento desses resultados e/ou que o sistema de pagamento por desempenho não estimule a continuidade de melhorias uma vez alcançadas as metas mínimas estabelecidas pela autoridade sanitária.

Christiansen et al. ${ }^{16}$ revisaram 36 artigos publicados sobre os impactos desses incentivos financeiros sobre a qualidade dos serviços prestados. Encontraram escassas avaliações de um impacto significativo e destacam a dificuldade de avaliar programas com desenhos muito distintos. Estimam, ainda, que a limitação do impacto desses incentivos financeiros é provavelmente devida, em alguns casos, ao fato de que um mesmo prestador possui distintos vínculos/contratos com órgãos financiadores diversos.

Outras limitações detectadas se referem à mensuração do desempenho: o número pequeno de pacientes portadores de determinados agravos (diabetes, p.ex.) tratado por cada médico; o fato de que os melhores médicos tratam, em ge- ral, dos casos mais complexos (o que leva à necessidade de avaliar o desempenho ajustado por risco) e, enfim, a complexidade de um processo de avaliação de desempenho, o qual, por sua vez, introduz custos adicionais ao sistema.

No início dos anos noventa, também nos EUA, algumas operadoras de planos de saúde (Health Maintenance Organizations - HMO) introduziram nos seus contratos com os prestadores incentivos pecuniários, para o atingimento de metas qualitativas, o sistema de benchmarking.

A experiência norte-americana de pagamento por desempenho qualitativo da atenção destinada a pacientes com infarto agudo do miocárdio, insuficiência cardíaca e pneumonia foi avaliada por Grossbart ${ }^{20}$. Nesse estudo, foram comparados quatro hospitais de uma organização sem finalidade de lucro pagos por desempenho com outros seis da mesma organização (grupo controle) que não aderiram a essa forma de remuneração. $\mathrm{O}$ artigo mostra que o grupo de hospitais pagos por desempenho exibiram maiores scores de qualidade nas três áreas clínicas estudadas, bem como um indicador global de qualidade mais satisfatório. Assim, o grupo teste conseguiu um indicador de qualidade $(89,7 \%)$ bastante superior ao do grupo controle $(85,6 \%)$. Vale mencionar que os indicadores de qualidade utilizados dizem respeito aos processos adotados pelos prestadores.

Estes dois estudos, bem como os de Giuffrida et al. ${ }^{21}$ e de Tannenbaum ${ }^{22}$, evidenciam que existe algum impacto do sistema de pagamento por desempenho sobre a qualidade da atenção à saúde, porém menor do que o desejável. Um fator que talvez explique, pelo menos em parte, esses resultados refere-se ao fato de que, na maior parte dos países que experimentaram esse modelo de alocação de recursos, os indicadores de desempenho qualitativo a partir dos quais são avaliados os prestadores referem-se, na sua maior parte, a processos da prática médico-assistencial e não a resultados sobre a saúde da população.

Vale mencionar a esse respeito Maynard ${ }^{4}$, ao afirmar que "o corolário da gestão do desempenho é a mensuração dos resultados sobre a saúde dos pacientes". Isso envolve, segundo o autor, não somente dados de mortalidade, mas também índices que retratam qualidade de vida dos pacientes antes e depois da assistencia à sua saúde. Ainda, como afirma Ortún ${ }^{23,24}$, é necessário introduzir medidas de resultado e de qualidade clínica para reduzir a distância entre eficácia e efetividade nos serviços de saúde. 


\section{Do volume ao valor}

A discussão internacional mais recente aponta para o fato de que a maior parte dos sistemas de alocação de recursos adotados na atualidade encorajam muito mais o aumento do volume dos serviços prestados do que o valor agregado, em termos de ganhos no estado da saúde da população, como destacado no NRHI Healthcare Payment Reform Summit (2008).

Nesse encontro, salientou-se a necessidade de dotar de mais responsabilidade os prestadores de serviços de saúde no que concerne ao aumento da qualidade e controle dos custos, sem penalizá-los quando tratam de pacientes mais complicados. E identificaram a necessidade de alocar recursos diferenciadamente segundo se tratasse da atenção a pessoas portadoras de condições crônicas (majoritariamente prestada pelo primeiro nível de atenção, embora de forma não exclusiva) ou se se tratasse de tratamentos efetuados em casos agudos. Note-se que pessoas portadoras de condições crônicas podem apresentar episódios agudos.

A atenção a episódios agudos deveria ser, segundo essa proposta, remunerada através do "pagamento por episódio de cuidado" (episodeof-care payment) - que consiste no estabelecimento de um único preço ou valor por todos os serviços requeridos para o tratamento desses casos, tais como infarto agudo do miocárdio, por exemplo. Para isso, seria alocado por paciente tratado um único valor global que incluiria a remuneração de todos os prestadores envolvidos no cuidado (SADT, honorários médicos, serviços hospitalares, etc.). Isso pressupõe a existência de uma entidade que receberia esse único pagamento e se responsabilizaria por remunerar todos os prestadores envolvidos, ou seja, requereria a existência de gestores de micro-sistemas constituidos por redes organizadas de prestadores de todos os níveis de atenção.

No que concerne à atenção primária destinada a pessoas portadoras de condições crônicas, propõe-se, diferentemente, a alocação de recursos por capitação, baseada em um valor per capita ajustado por risco, para que o prestador seja responsável por mantê-los em boas condições de saúde (e evitar internações), segundo os princípios da atenção centrada no paciente (patientcentered medical home).

A proposta ainda contempla o estímulo aos pacientes no sentido de utilizarem prestadores mais eficientes, ou seja, que prestem uma assistência de boa qualidade a um menor custo do que outros, $\mathrm{o}$ qual reforça a necessidade de constituir redes integradas de prestadores de serviços de saúde.

Nessa mesma direção se encontra a proposta da alocação de recursos por caso (crônico) gerido, no contexto da gestão integrada da doença. $\mathrm{Na}$ literatura portuguesa, esse modelo de alocação de recursos é denominado "pagamento por preço compreensivo" 25 que, segundo esses autores, corresponde a um valor médio por paciente por um determinado período de tempo, que "engloba o conjunto de atos clínicos, medicamentos e outras atividades consideradas essenciais para uma adequada prestação de cuidados". Todavia, a efetivação da alocação de recursos depende, nessa proposta, do cumprimento de parâmetros de qualidade e segurança do paciente, aferidos através de um conjunto de indicadores de resultados.

Esse modelo pressupõe a existência de um prestador responsável pela gestão da linha de cuidados requeridos por cada grupo de pacientes e, assim, responsável pelo pagamento a outros prestadores situados em distintos níveis de atenção. No caso português, este modelo começa a ser experimentado através da instituição dos Centros de Elevada Diferenciação (CED), entendidos como unidades que desenvolvem competências clínicas e de investigação e estruturam o cuidado integral dos pacientes. O modelo requer, ainda, a estruturação de redes integradas de serviços, a serem geridas ou contratualizadas por essos centros.

\section{Considerações finais}

Uma apreciação mais global da experiência internacional mostra as seguintes tendências: os métodos tradicionais de alocação de recursos foram substituídos por sistemas mistos ou complementados pelo ajuste por desempenho; a alocação de recursos ajustada por desempenho requer, para ser mais eficaz, a adoção de indicadores de desempenho que, para além de medirem processos assistenciais e de gestão (essencialmente à eficiência e qualidade), se refiram predominantemente a resultados sobre a saúde da população, ou seja, à efetividade dos serviços de saúde.

Nesse sentido, percebe-se uma tendência a adotar sistemas de alocação de recursos destinados a centros de atenção primária responsáveis pela saúde da população de um dado território que, se necessário, contratarão serviços de outros níveis de atenção. Em decorrência disso, concebe-se a alocação de recursos por linhas de cuidado, por gestão da doença ou, nos termos por- 
tugueses, por "preço compreensivo", destinada aos responsáveis por essa gestão do cuidado, geralmente em mãos da atenção primária.

Dessa forma, propugnam-se modalidades distintas de alocação de recursos para a atenção primária (em base à capitação, ajustada por ris- co, por desempenho ou por algum outro critério) e para os hospitais, a serem remunerados por orçamento global ajustado por desempenho (qualitativo e quantitativo) ou por pagamento prospectivo por procedimento, dependendo do país de que se trate.

\section{Referências}

1. Wennberg JE, Gittelsohn A. Variations in medical care among small areas. Science 1973; 246(4):100111.

2. Ugá MAD. As propostas de reforma dos sistemas de saúde no contexto do ajuste macroeconômico [tese]. Rio de Janeiro: Instituto de Medicina Social da UERJ; 1997.

3. BMJ. BMJ Clinical Evidence Handbook. London: BMJ Publishing; 2007.

4. Maynard A. Payment for Performance (P4P): International experience and a cautionary proposal for Estonia. Health Financing Policy Paper. Geneva: World Health Organization (WHO), Division of Country Health Systems; 2008.

5. Ugá MAD. Sistemas de repasses financeiros a unidades de assistência à saúde: uma proposta preliminar para a rede pública brasileira. In: Ugá MAD. O financiamento da saúde no Brasil. Brasília: Organização Pan-Americana da Saúde, Representação do Brasil; 1994; p. 72-98. (Série Economia e Financiamento, n. 4).

6. Ugá MAD. O Sistema de Pagamento Prospectivo por Procedimento: a Experiência Norte-Americana. Cadernos FUNDAP 1996; 38(19):170-185.

7. Gay EG, Kronenfeld J, Baker S, Amidon R. An appraisal of organizational response to fiscally constraining regulation: the case of hospitals and DRGs. J Health Soc Behav. 1989; 30(1):41-55.

8. Chulis G. Assessing Prospective Payment. Med Care Rev 1991; 48(2):167-206.

9. Feinglass J, Holloway MD. The initial impact of the Medicare Prospective Payment System on U.S. health care: a review of literature. Med Care Rev 1991; 48(1):91-115.

10. Hornbrook MC, Berki S. Practice Mode and Payment Method: effects on use, costs, quality and access. Med Care 1985; 23(5):484-511.

11. Carter GM, Newhouse JP, Relles A. How much change in the Case Mix Index is DRG Creep? J Health Econ 1990; 9(4):411-428.

12. Guterman S, Dobson A. Impact of the Medicare Prospective Payment System for Hospitals. Health Care Financ Rev. 1986; 7(6):97-114.

13. Cylus J, Irwin R. The challenges of hospital payment systems. Geneva: World Health Organization; 2010.

14. Docteur E. More value for money: improving efficiency in OECD health systems. Berlin: OECD; 2004.
15. Kazandjian V. Pay-for-performance in health care: the natural evolution of performance measurement and community expectations. Rev Portuguesa de Saúde Pública 2010; 9:117-128.

16. Christianson J, Leatherman S, Sutherland K. Financial incentives, healthcare providers and quality improvements. A review of evidence. London: The Health Foundation; 2007.

17. Afonso PB. Contratualização em ambiente de cuidados de saúde primários. Rev Portuguesa de Saúde Pública 2010; 9:59-64.

18. Campbell SM, Reeves D, Kontopantelis E, Sibbald B, Roland M. Effects of pay for performance on the quality of primary care in England. $N$ Engl J Med 2009; 361(4):368-378.

19. Peiró S. Comentario. Gestión Clínica y Sanitaria 2009; 11(2):64.

20. Grossbart S. What's the Return? Assessing the Effect of "Pay-for-Performance" Initiatives on the Quality of Care Delivery. Med Care Res Rev 2006; 63(1):29-48.

21. Giuffrida A, Gosden T, Forland F, Kristiansen I, Leese B, Pedersen L, Sutton M. Target payments in primary care: effects on professional practice and health care outcomes (Cochrane Rewiew). Oxford: The Cochrane Library; 2000.

22. Tanenbaum S. Pay for Performance in Medicare: Evidentiary Irony and the Politics of Value. J Health Polit Policy Law 2009; 34(5):47-60.

23. Ortún V. De la evaluación a la Gestión: acortar la brecha entre eficacia y efectividad. Ekonomiaz 2005; 60(1):58-75.

24. Ortún V. El Buen Gobierno Sanitario. Economía y Gestión Sanitaria. Madrid: Springer Healthcare Communications; 2009.

25. Escoval A, Coelho A, Diniz J, Rodrigues M, Moreira F, Espiga P. Gestão integrada da doença: uma abordagem experimental de gestão em Saúde. Rev Portuguesa de Saúde Pública 2010; 9:105-116.

Artigo apresentado em 21/06/2011

Aprovado em 14/07/2011

Versão final apresentada em 04/08/2011 